\title{
Rapid Tempering: Opportunities and Challenges
}

\author{
V.K. Euser, A.J. Clarke, and J.G. Speer
}

(Submitted June 1, 2020; in revised form June 11, 2020; published online July 14, 2020)

\begin{abstract}
Rapid tempering involves shorter times and faster heating rates compared to conventional tempering. Utilizing rapid tempering via industrial processes, such as induction heating, not only offers the opportunity for improved mechanical properties, but also for reduced processing times and energy costs. The current study demonstrates an improvement in toughness with rapid tempering by examining impact toughness at a constant tempering parameter for short-time $(1 \mathrm{~s})$ and conventional $(3600 \mathrm{~s})$ tempering treatments of 4340 steel. Fracture behavior is found to complement the observed toughness behavior, where higher impact energy conditions are associated with a greater percentage of ductile fracture. The role of the Hollomon-Jaffe tempering parameter, as well as the use of hardness as a metric to characterize degree of tempering, is discussed in light of the results comparing rapid and conventional tempering conditions. This study indicates promising mechanical properties associated with short-time tempering, especially within the tempered martensite embrittlement (TME) regime, and exposes challenges associated with predicting performance strictly based on hardness when rapid tempering is utilized.
\end{abstract}

Keywords dynamic, heat treatment, impact toughness, mechanical, rapid tempering, steel, tempered martensite, tempered martensite embrittlement

\section{Introduction}

The Hollomon-Jaffe tempering parameter has long been used to relate time and temperature during tempering of steel alloys, where relative hardness serves as the basis of the relationship (Ref 1). Since the introduction of the tempering parameter, it has been successfully applied to a variety of steel alloys across a wide range of time-temperature combinations (Ref 1-7). Adjustments to the original formulation have been developed to incorporate steel composition variations (Ref 7) and non-isothermal tempering (Ref 5). The tempering parameter, typically in its modified non-isothermal form, has been successfully applied in several short-time tempering studies

This paper is the 2019 HTS-Bodycote Best Paper in Heat Treating. The ASM Heat Treating Society established the Best Paper in Heat Treating Award in 1997 to recognize a paper that represents advancement in heat-treating technology, promotes heat treating in a substantial way, or represents a clear advancement in managing the business of heat treating. The award, endowed by Bodycote Thermal Process-North America, is open to all students, in full-time or part-time education, at universities (or their equivalent) or colleges. It also is open to those students who have graduated within the past three years and whose paper describes work completed while an undergraduate or postgraduate student. The subject matter of the paper is required to cover any aspect of research or development applied to the fields of heat treatment, surface engineering, hot isostatic pressing (hip), metallurgical coatings, testing, or materials processing, including any production process, information technology, or relevant management or business issues.

V.K. Euser, Los Alamos National Laboratory, Los Alamos, NM; and Colorado School of Mines, Golden, CO; and A.J. Clarke and J.G. Speer, Colorado School of Mines, Golden, CO. Contact e-mail: ginny.euser@lanl.gov.

(Ref 3, 5, 8, 9), although Furuhara et al. (Ref 10) indicated a significant deviation in the hardness-tempering parameter relationship for tempering conditions associated with rapid heating rates and a $0 \mathrm{~s}$ hold time. A zero second treatment involves heating to a specific temperature, followed by immediate cooling. Previous studies by the current authors (Ref 11,12 ) have brought into question the applicability of the Hollomon-Jaffe tempering parameter for rapid tempering treatments - not due to a breakdown in the relationship between tempering parameter and hardness, but rather due to the observation of varying properties at a constant hardness value obtained through different heat treatment parameters. The current study further explores the toughness and fracture properties associated with rapid tempering, and discusses the role of hardness as a tempering indicator in the context of shorttime tempering. In addition, an in-depth overview of the methodology adopted in designing the tempering matrix and the approach for accurately executing rapid tempering treatments are presented.

\section{Experimental Methods}

\subsection{Materials and Thermal Processing}

A 4340 steel, with the composition listed in Table 1, was utilized in the present study. The material was received in the form of $12.7 \mathrm{~mm}$ (0.5 in) thick, annealed plates. Prior to heat treatment, rectangular Charpy blanks $(90 \times 11 \times 11 \mathrm{~mm})$ were machined from the original plates. Blanks were austenitized for $1 \mathrm{~h}$ at $845^{\circ} \mathrm{C}$, and subsequently oil-quenched. Tempering temperatures ranging from 200 to $400{ }^{\circ} \mathrm{C}$ were chosen for conventional (3600 s) tempering treatments. Conventional tempers were carried out in a nitrate salt bath, followed by water quenching. The Hollomon-Jaffe tempering parameter was utilized to determine appropriate tempering temperatures that would provide an equivalent degree of tempering for the $1 \mathrm{~s}$ conditions. The tempering parameter is represented as: 
Table 1 Chemical composition of AISI 4340 research material (wt.\%)

\begin{tabular}{lcccccccccccc}
\hline $\mathbf{C}$ & $\mathbf{M n}$ & $\mathbf{S i}$ & $\mathbf{N i}$ & $\mathbf{C r}$ & $\mathbf{M o}$ & $\mathbf{N b}$ & $\mathbf{V}$ & $\mathbf{A l}$ & $\mathbf{S}$ & $\mathbf{P}$ & $\mathbf{C u}$ \\
\hline 0.41 & 0.71 & 0.25 & 1.76 & 0.75 & 0.26 & 0.005 & 0.047 & 0.008 & 0.001 & 0.009 & 0.14 \\
\hline
\end{tabular}

Table 2 Time-Temperature Tempering Matrix

\begin{tabular}{lrrrrr}
\hline Time, $\mathbf{s}$ & \multicolumn{5}{c}{ Temperature, ${ }^{\circ} \mathbf{C}$} \\
\hline 3600 & 200 & 250 & 300 & 350 & 400 \\
1 & 305 & 366 & 427 & 489 & 550 \\
TP (approximate) & 9000 & 10,000 & 11,000 & 12,000 & 13,000 \\
\hline
\end{tabular}

$T P=T(\log (t)+c)$

where $T$ is absolute temperature, $t$ is time in seconds, and $c$ is a material-dependent constant. A $c$-value of 16 was used in the current study, as Hollomon and Jaffe concluded that a $c$-value of 16 (for time in seconds) resulted in satisfactory results for plain carbon and alloy steels with carbon contents ranging from 0.25 to $0.4 \mathrm{wt}$ pct (Ref 1$)$. The tempering temperatures applicable to the $1 \mathrm{~s}$ tempering times were determined by utilizing the TPS of the conventional tempering conditions and solving for $T$ in Eq 1. The resulting time-temperature combinations used in the current study are shown in Table 2. Shorttime tempering treatments were carried out in a Gleeble 3500 followed by quenching with helium, where heating and cooling rates ranged from approximately $800-1000$ and $30-60{ }^{\circ} \mathrm{C} / \mathrm{s}$, respectively. Figure 1 shows an example of a thermal profile achieved for a $1 \mathrm{~s}-305{ }^{\circ} \mathrm{C}$ treatment compared to the ideal isothermal profile. The differences between experimental and "ideal" thermal profiles at high temperatures are magnified in Fig. 1b.

An adapted Hollomon-Jaffe tempering parameter, introduced by Semiatin et al. (Ref 5), was used to quantitatively evaluate differences in experimental and ideal thermal profiles, such as those shown in Fig. 1(a) and (b). The method introduced by Semiatin et al. accounts for non-isothermal tempering that primarily occurs during heating and cooling. The non-isothermal formulation of the tempering parameter is:

$T P=T_{i}\left(c+\log \Delta t_{i}\right)$

where $c$ is the $c$-value, $T_{i}$ and $t_{i}$ represent the temperature and time, respectively, and $\Delta t_{i}$ the time step over which the experimental temperature is evaluated. The TP values are then integrated over all recorded time steps to represent an "equivalent isothermal tempering parameter", from which an "equivalent isothermal tempering time" can be extracted. The largest deviation between the desired tempering time and the calculated isothermal tempering time was on the order of $0.2 \mathrm{~s}$, while the majority of points fell within $0.1 \mathrm{~s}$ of the target tempering time. In the context of tempering parameter, the largest time deviation corresponds to $65 \mathrm{TP}$ units, less than 1 pct of the overall TP of the utilized tempering treatments.

\subsection{Mechanical Testing}

Charpy impact test specimens were machined from the heattreated Charpy blanks per ASTM standard E23 (Ref 13). Charpy testing was conducted at $-200,-100,0,25$ (room temperature), 100 , and $200{ }^{\circ} \mathrm{C}$. All testing was performed in accordance with ASTM E23, with the exception of specimens tested at $200{ }^{\circ} \mathrm{C}$. A furnace, along with a surrounding thermal mass, was used to achieve testing temperatures of $200{ }^{\circ} \mathrm{C}$, where the samples were determined to reach thermal equilibrium after 5 min. Vickers microhardness testing was performed on tempered specimens prepared via standard metallographic practices and final polishing to a $6 \mu \mathrm{m}$ finish. A LECO MHT200 indenter was used with a $500 \mathrm{gf}$ load and $10 \mathrm{~s}$ dwell time. Fifteen indentations were made per tempering condition. Rockwell C values were obtained from Vickers hardness values by using the standard hardness conversion equations outlined in ASTM E120 (Ref 14). Due to the nature of resistive heating utilized in the Gleeble 3500, a thermal gradient was produced along the length of the specimens, resulting in a temper gradient. Therefore, care was taken to evaluate hardness and impact toughness properties associated with the appropriately tempered area of the specimens.

\subsection{Fractography: Shear Lip Area and Brittle Point Fraction}

In ASTM E23 (Ref 13), the methodology for measuring shear fracture area of Charpy impact specimens is applicable to carbon steels that undergo a distinct ductile to brittle transition, resulting in a clear region of cleavage fracture. However, materials that exhibit quasi-cleavage fracture, such as those explored in the present study, complicate fracture surface interpretation, as there are not optically distinct regions associated with cleavage and fibrous fracture. Thus, two methodologies, focused on macroscopic and microscopic fracture behavior, were developed to examine the Charpy fracture surfaces in the current work.

Macroscopic fracture behavior was assessed by quantifying the area of the fracture surface associated with the formation of shear lips. This is distinctly different than the shear fracture area measurement in ASTM E23 (Ref 13), as only the shear lip area is quantified, rather than the entire fracture surface area associated with fibrous ("shear") fracture. Charpy shear lip area was determined by examining macroscopic images of Charpy fracture surfaces using ImageJ (Ref 15), an image analysis software. Images were evaluated by outlining regions identified as shear lips, and subsequently thresholding the image to represent shear lips as black, and all other regions as white. The area percentage associated with shear lips was then determined using the particle analyzer function available in ImageJ. Figure 2 shows an example of the shear lip area measurement procedure implemented in the present work. Some conditions, like the one shown in Fig. 2, exhibited a small, shear region associated with final fracture that was included in the shear lip area determination. 


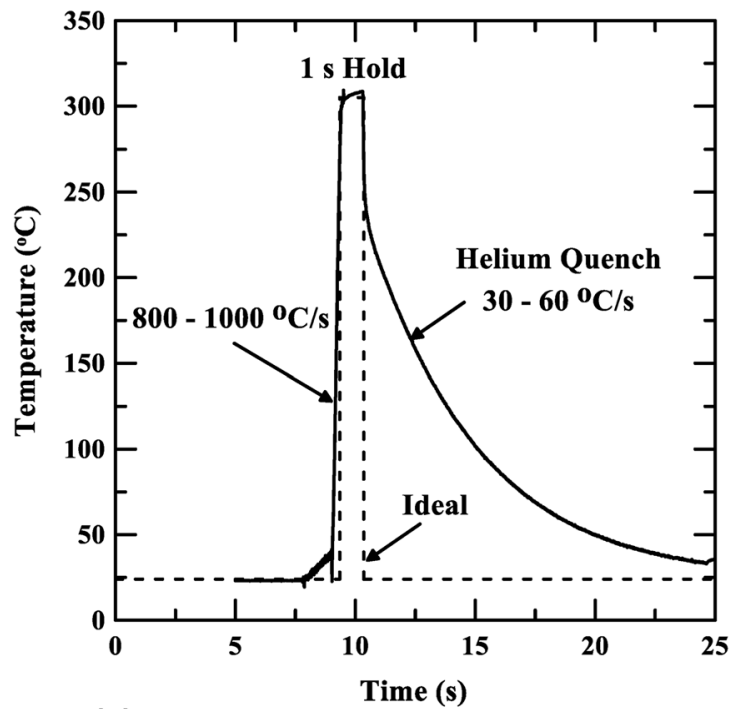

(a)

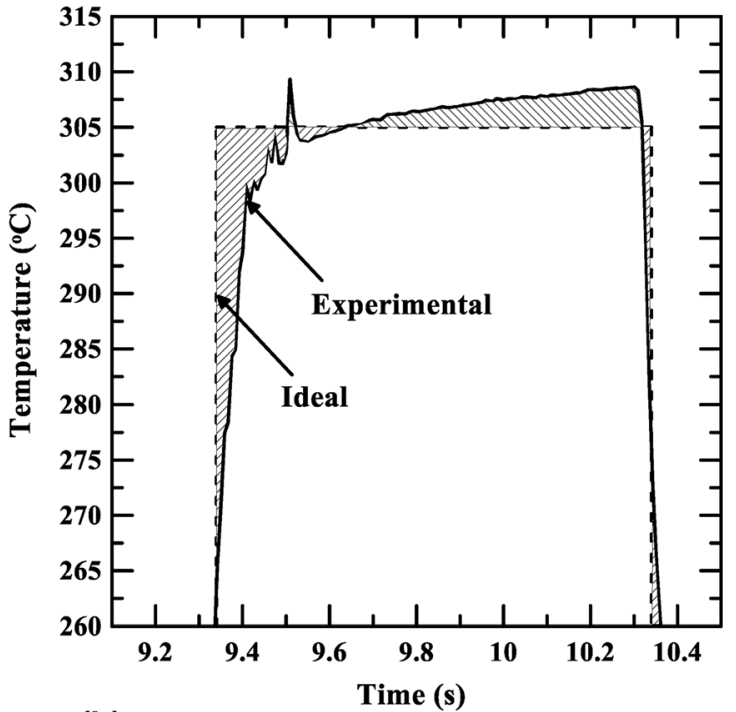

(b)

Fig. 1 Comparison of "ideal" and actual thermal profiles during Gleeble tempering at the (a) full temperature and time scale, as well as the (b) high temperature regime

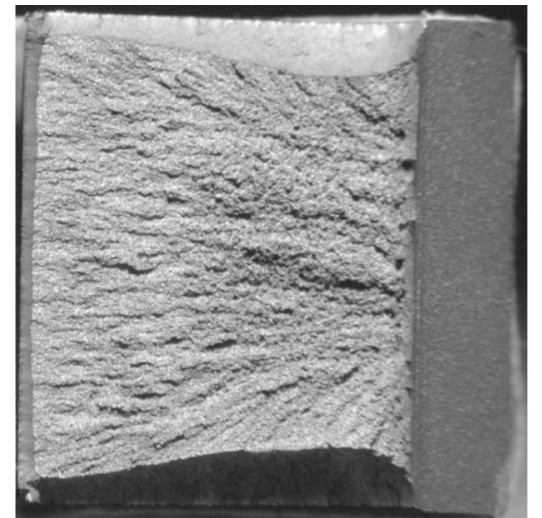

(a)

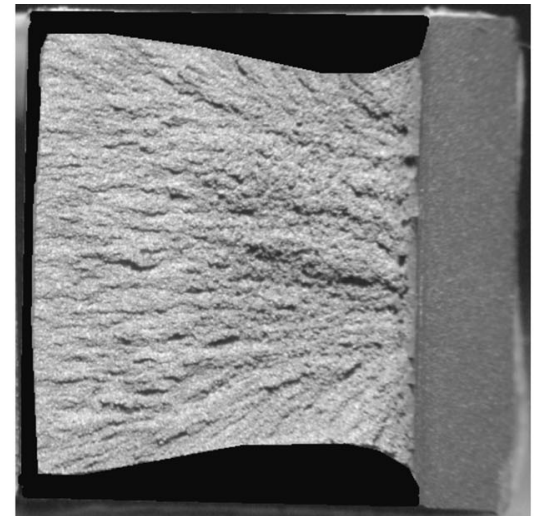

(b)

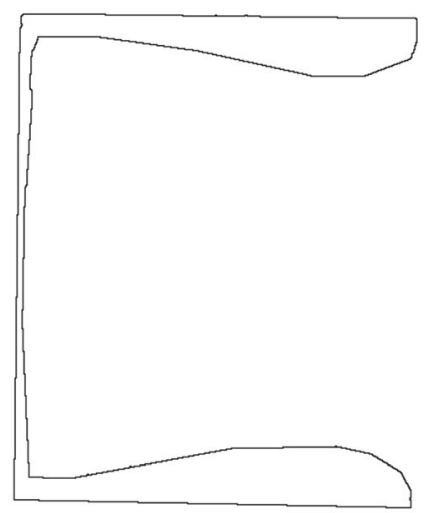

(c)

Fig. 2 Example of shear lip area evaluation including (a) original image of fracture surface, (b) filled in shear lip area, and (c) resultant outlined area evaluated by the particle analyzer feature in ImageJ. Determined shear lip area of sample corresponds to $19 \%$

While the macroscopic evaluation of shear lip area gives some indication of the fracture characteristics, the behavior within the flat regions of the Charpy fracture surface is not captured within this metric. Thus, the microscopic fracture behavior within the flat areas of the Charpy impact specimens was evaluated using a JEOL 7000 field emission scanning electron microscope (FESEM). Micrographs were taken at consistent positions along the centerline (perpendicular to the notch) of all examined conditions. To quantify the microscopic fracture behavior of the various conditions, a "brittle point fraction" parameter was developed and used. Much of the procedure was modeled after the point counting method for determining phase volume fraction (Ref 16). Brittle point fraction was determined by overlaying a 100-point grid on micrographs of the Charpy V-notch fracture surfaces obtained at a consistent position and magnification. At each line intersection (point), the behavior was categorized as consistent with either cleavage (brittle) or microvoid coalescence (ductile) fracture. The brittle point fraction (BPF) is defined here as the number of points characterized as cleavage fracture divided by the total number of counted points. Transition regions were tallied as a third entity and then split between ductile and brittle fracture after point counting of the fractograph was complete.

\section{Results and Discussion}

Figure 3 depicts hardness as a function of the HollomonJaffe tempering parameter for the time-temperature combinations outlined in Table 2. The conventional and short-time tempering conditions exhibit comparable hardness values at a given tempering parameter. To account for any small differences in hardness at nominally equivalent tempering parameters, some property comparisons will be presented as a function of the tempered hardness.

Charpy impact absorbed energy is displayed as a function of testing temperature for time-temperature conditions associated with 9000, 11,000, and 13,000 TPs in Fig. 4. At 9000 TP, the transition temperature curves of the two tempering conditions 
overlap. As tempering parameter increases to 11,000 and 13,000 TP, the impact transition curves become more distinct, where short-time conditions exhibit higher impact energies across the full range of explored testing temperatures. Figure 5 displays shear lip area measurements corresponding to the conditions in Fig. 4. Again, the 1 and 3600 s conditions exhibit similar behavior at a tempering parameter of $9000 \mathrm{TP}$. However, at tempering parameters of 11,000 and 13,000 , the short-time conditions exhibit larger percentages of shear lip area.

Charpy fracture surfaces from room temperature $\left(25^{\circ} \mathrm{C}\right)$ test specimens associated with 11,000 and 12,000 TPs were assessed to quantify the microscopic fracture behavior. Fractographs corresponding to 1 and $3600 \mathrm{~s}$ at $12,000 \mathrm{TP}$ are displayed in Fig. 6. A mixture of ductile, microvoid coalescence and transgranular cleavage (with respect to prior

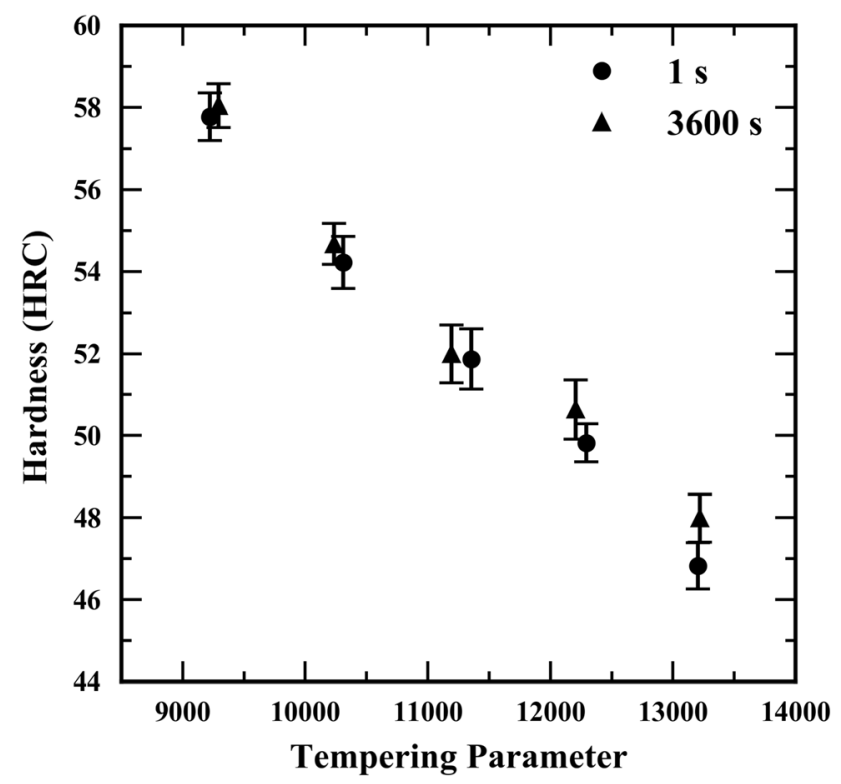

Fig. 3 Hardness (HRC) as a function of tempering parameter for 1 and $3600 \mathrm{~s}$ conditions. Displayed error bars correspond to the standard deviation associated with 15 measurements for each timetemperature condition austenite grains) is observed for both tempering conditions, consistent with TME fracture behavior often reported in literature when impurity elements such as phosphorus do not play an embrittling role (Ref 17-25). The prevalence of cleavage fracture was quantified via BPF measurements. The results are presented in Table 3 , where short-time conditions exhibit a greater degree of ductile fracture (lower BPF) for both tempering parameters explored. While ductile fracture increases markedly from 11,000 to $12,000 \mathrm{TP}$ for the short-time condition, the conventional treatment exhibits a constant BPF with increasing tempering parameter. The impact energy and fractography results consistently indicate improved toughness as a result of short-time tempering within the TME regime.

The present results indicate an improvement in toughness with short-time tempering across a wide range of testing temperatures. The impact toughness transition curves associated with rapid tempering shift to lower testing temperatures and higher impact energies compared to conventional tempering. Additionally, improvement in toughness properties with rapid tempering is corroborated by the observed fracture behaviors. The shear lip area and toughness curves correspond well, where higher shear lip percentages correlate with increased impact energies. Rapid tempering conditions also exhibit more ductile behavior at the microscopic scale, as indicated by the BPF trends.

The hardness results in Fig. 3 indicate the Hollomon-Jaffe tempering parameter is suitable for relating time, temperature, and resulting hardness in the context of short-time tempering in 4340; however, corresponding toughness and fracture behavior do not follow the TP methodology. That is, toughness varies across conditions that have equivalent tempering parameter and hardness, with better toughness observed for conditions involving shorter times at higher temperatures. Historically, the aim of tempering parameter studies has been to develop a predictable relationship between tempering parameter and hardness, with the underlying assumption that other key properties would closely follow tempered hardness (Ref 1). This practice has been largely successful within a given alloy system, assuming similar austenitization and prior processing conditions. However, the current study reveals significant variation in toughness at a given tempered hardness for the same alloy composition and prior processing routes. If hardness does not accurately reflect toughness (and potentially strength

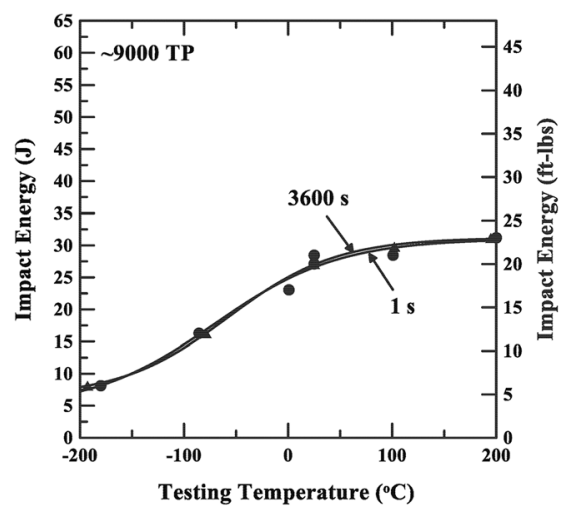

(a)

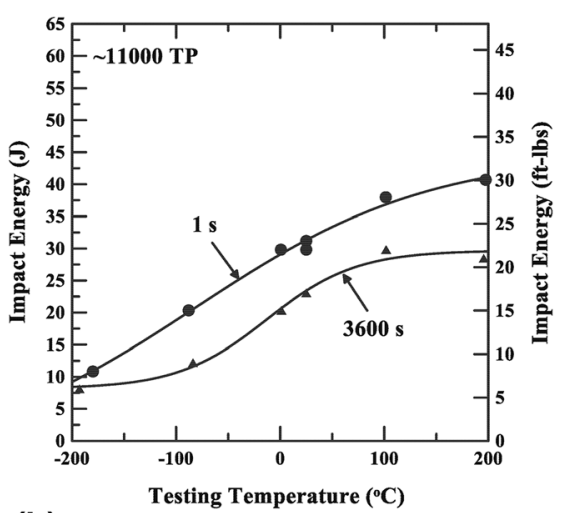

(b)

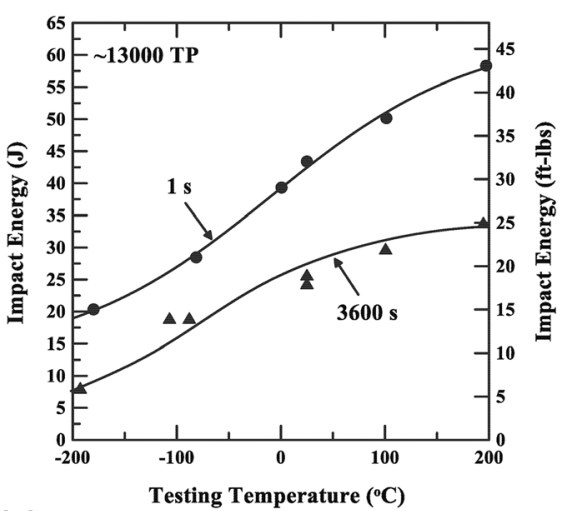

(c)

Fig. 4 Charpy impact energy as a function of testing temperature for short-time and conventional conditions and tempering parameters of (a) 9000, (b) 11,000, and (c) 13,000. Hardness values for the 1 and $3600 \mathrm{~s}$ tempering times are 57.9 and 58.1 HRC, 52.1 and 52.2 HRC, and 47.1 and $48.4 \mathrm{HRC}$ for the $9000,11,000$, and $13,000 \mathrm{TP}$ conditions, respectively 


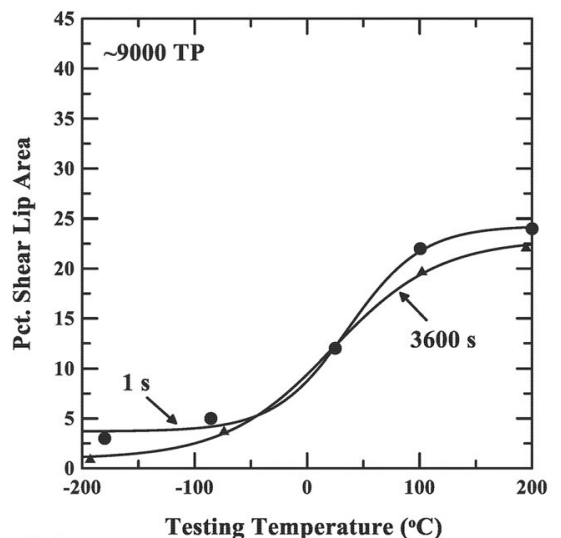

(a)

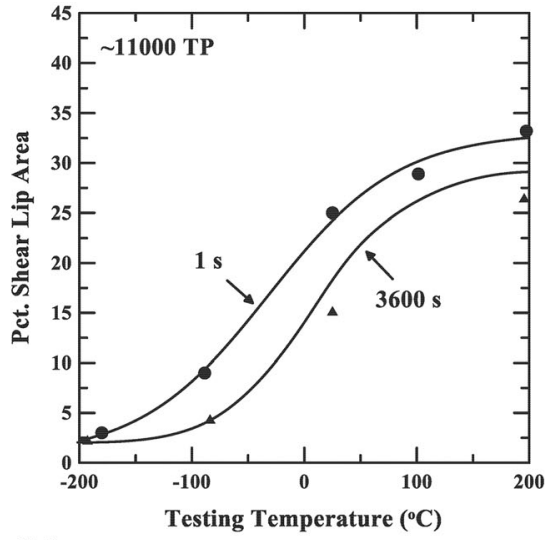

(b)

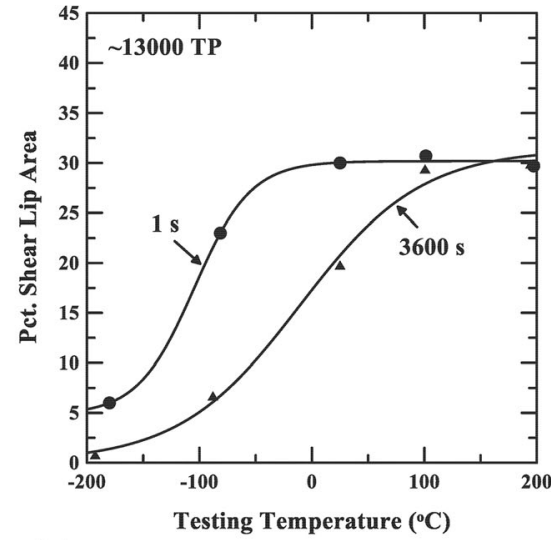

(c)

Fig. 5 Percent shear lip area as a function of testing temperature for short-time and conventional conditions and tempering parameters of (a) 9000, (b) 11,000, and (c) 13,000. Hardness values for the 1 and $3600 \mathrm{~s}$ tempering times are 57.9 and 58.1 HRC, 52.1 and 52.2 HRC, and 47.1 and $48.4 \mathrm{HRC}$ for the $9000,11,000$, and 13,000 TP conditions, respectively

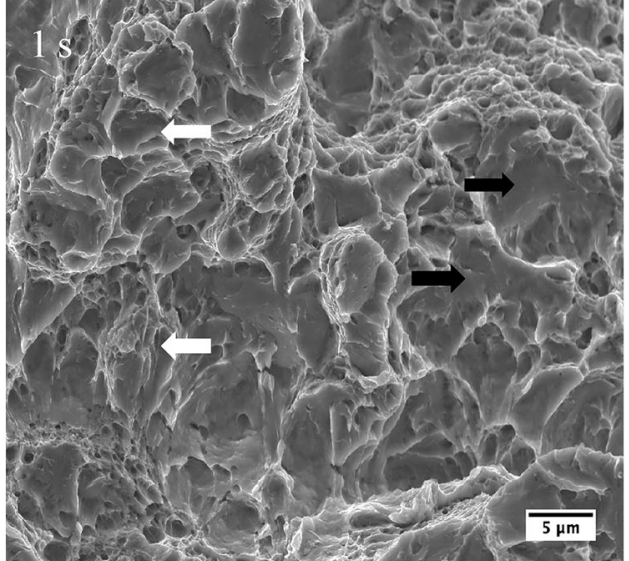

(a)

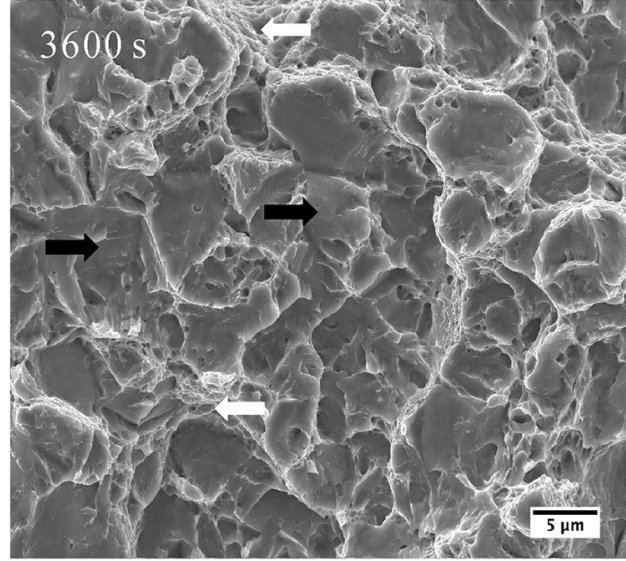

(b)

Fig. 6 Representative fractographs of Charpy impact specimens tested at room temperature associated with (a) short-time and (b) conventional conditions at a tempering parameter of 12,000. Micrographs were obtained at consistent positions ( $4 \mathrm{~mm}$ from the notch) along the centerline (perpendicular to the notch) of the fracture surface. White and black arrows denote examples of regions considered to represent fibrous (ductile) and cleavage (brittle) fracture, respectively

Table 3 Brittle point fraction from room-temperature-tested Charpy fracture surfaces

\begin{tabular}{lccc}
\hline Tempering parameter & Tempering time, $\mathbf{s}$ & Tempering temperature, ${ }^{\circ} \mathbf{C}$ & Average brittle point fraction \\
\hline 11,000 & 1 & 427 & 0.38 \\
11,000 & 3600 & 300 & 0.52 \\
12,000 & 1 & 489 & 0.16 \\
12,000 & 3600 & 350 & 0.51 \\
\hline
\end{tabular}

(Ref 11, 12)) changes between short-time and conventional tempering for a given alloy, then it may be appropriate to ask - what purposes does a hardness-based tempering parameter serve in the context of short-time tempering?

Tempering is typically associated with decreasing hardness and a corresponding increase in toughness. However, this relationship between hardness and toughness is known to break down in the presence of an embrittling mechanism such as TME. Figure 7 shows the impact toughness results as a function of tempered hardness for the conventional (3600 s) and short-time $(1 \mathrm{~s})$ tempers. The $3600 \mathrm{~s}$ condition exhibits the hardness-toughness behavior typically associated with TME, where impact toughness decreases with decreasing hardness within a specific regime. This decrease in toughness results in a toughness trough, the depth of which is often used to assess the severity of TME. In contrast, the short-time condition exhibits a more consistent increase in toughness with decreasing tempered hardness, indicating only slight effects that may relate to TME 


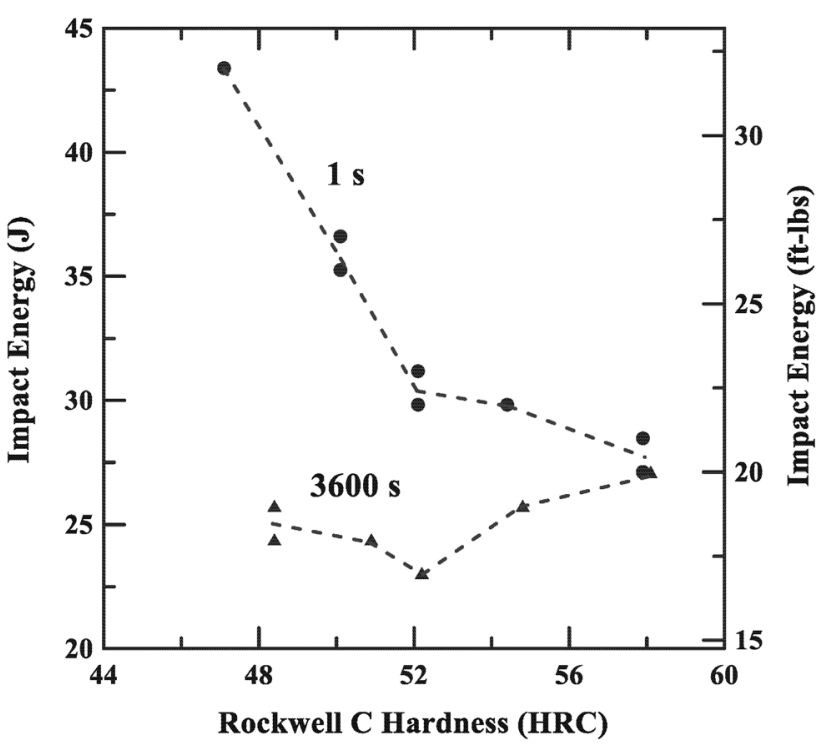

Fig. 7 Room-temperature impact toughness as a function of hardness for tempering times of 1 and $3600 \mathrm{~s}$. Two samples were tested per condition, one data point for a given condition signifies that the two results were identical

mechanisms. The discrepancy in toughness properties between short-time and conventional conditions at an equivalent hardness may, therefore, be connected to differences in TME behavior.

Tempered martensite embrittlement is often attributed to specific changes in microstructure, such as retained austenite decomposition ( $\operatorname{Ref} 19,26)$, cementite precipitation (Ref 19), and/or cementite coarsening (Ref 27, 28). The evident difference in toughness behavior, and more specifically TME severity, between conventional and rapid tempering conditions suggests a corresponding difference in microstructural evolution. Since the progression of tempering is fundamentally considered in the context of microstructural development (Ref 29), the failure of hardness measurements to capture fine microstructural changes presents a potential issue in using hardness as the principal tempering metric. This is particularly true if the small microstructural variations, undetected by hardness, greatly affect other mechanical properties such as toughness or strength, as the relationship between tempering and resulting mechanical properties is typically the driver for tracking tempering progression. The short-time tempering results of the present study indicate that the insensitivity of hardness to certain microstructural changes may be relevant in the context of rapid tempering, particularly within the TME regime. This behavior has important implications for rapidly tempered components, where new relationships between processing (tempering time/temperature), properties (hardness), and performance may need to be explored.

\section{Conclusions}

Rapid tempering of 4340, achievable through processes such as induction heating, was shown to improve impact toughness across all explored testing temperatures compared to conventional tempering at an equivalent hardness. Shear lip and BPF measurements complemented the observed toughness behavior, in which more ductile (shear) fracture was observed in association with higher impact toughness. Rapid tempering presents an opportunity for enhanced mechanical properties via novel heat treatment routes; however, the use of hardness to describe tempering degree should be used with caution when employing short-time tempering. Large variations in toughness performance at a given tempered hardness may present difficulties in tracking tempering progression and resultant mechanical properties during the practical implementation of heat treating. New relationships between processing and performance must be developed in the context of short-time tempering.

\section{Acknowledgments}

The authors gratefully acknowledge the support of the Advanced Steel Processing and Products Research Center (ASPPRC), an industry/university cooperative research center at the Colorado School of Mines in Golden, CO, USA. VKE gratefully acknowledges support by the U.S. Department of Energy through the Los Alamos National Laboratory (LANL) during the preparation of this manuscript. LANL is operated by Triad National Security, LLC, for the National Nuclear Security Administration of U.S. Department of Energy (Contract No. 89233218CNA000001). LANL is also thanked for supplying the steel studied in this work.

\section{References}

1. J.H. Hollomon and L.D. Jaffe, Time-Temperature Relations in Tempering Steel, Trans. AIME, 1945, 162, p 223-249

2. A. Nakashima and J.F. Libsch, Effect of Induction on 500 Embrittlement, Trans. ASM, 1961, 53, p 753-764

3. J.F. Libsch and A.E. Powers, Rapid Tempering by Induction Heating, Met. Prog., 1950, 58, p 176-180

4. S. Murphy and J.H. Woodhead, An Investigation of the Validity of Certain Tempering Parameters, Metall. Trans., 1972, 3(March), p 727735

5. S.L. Semiatin, D.E. Stutz, and T.G. Byrer, Induction Tempering of Steel: Part I. Development of an Effective Tempering Parameter, J. Heat Treat., 1985, 4(1), p 39-46

6. R.A. Grange, C.R. Hribal, and L.F. Porter, Hardness of Tempered Martensite in Carbon and Low-Alloy Steels, Metall. Trans., 1977, 8(11), p 1775-1785

7. R.A. Grange and R.W. Baughman, Hardness of Tempered Martensite in Carbon and Low Alloy Steels, Trans. ASM, 1956, 48, p 165-197

8. A. Nagao, K. Hayashi, K. Oi, S. Mitao, and N. Shikanai, Refinement of Cementite in High Strength Steel Plates by Rapid Heating and Tempering, Mater. Sci. Forum, 2007, 539, p 4720-4725. https://doi. org/10.4028/www.scientific.net/MSF.539-543.4720

9. C. Revilla, B. López, and J.M. Rodriguez-Ibabe, Carbide Size Refinement by Controlling the Heating Rate during Induction Tempering in a Low Alloy Steel, Mater. Des., 2014, 62, p 296-304. https://doi.org/10.1016/j.matdes.2014.05.053

10. T. Furuhara, K. Kobayashi, and T. Maki, Control of Cementite Precipitation in Lath Martensite by Rapid Heating and Tempering, ISIJ Int., 2004, 44(11), p 1937-1944. https://doi.org/10.2355/isijinternatio nal.44.1937

11. V.K. Judge, J.G. Speer, K.D. Clarke, K.O. Findley, and A.J. Clarke, Rapid Thermal Processing to Enhance Steel Toughness, Sci. Rep., 2018, 8(January), p 1-6. https://doi.org/10.1038/s41598-017-18917-3

12. V.K. Euser, D.L. Williamson, K.D. Clarke, K.O. Findley, J.G. Speer, and A.J. Clarke, Effects of Short-Time Tempering on Impact Toughness, Strength, and Phase Evolution of 4340 Steel Within the Tempered Martensite Embrittlement Regime, Metall. Mater. Trans. A, 2019, 50(August), p 3654-3662. https://doi.org/10.1007/s11661-019-052714 
13. ASTM Standard E23, Standard Methods for Notched Bar Impact Testing of Metallic Materials, ASTM International, West Conshohocken, PA, 2018, p 2018

14. ASTM E140-12b(2019)e1, Systematic Manual Point Count, ASTM International, West Conshohocken, PA, 2018

15. W.S. Rasband, Image J, U.S. National Institutes of Health, Bethesda, Maryland, https://imagej.nih.gov/ij/

16. ASTM E562, Standard Test Method for Determining Volume Fraction by Systematic Manual Point Count, ASTM International, West Conshohocken, PA, 2018, p 2018https://doi.org/10.1520/E056 $2-11.2$

17. B.V.N. Rao and G. Thomas, Retained Austenite and Transgranular Tempered Martensite Embrittlement, in 4th International Conference on Fracture, 1977, p 1-12

18. G. Thomas, Retained Austenite and Tempered Martensite Embrittlement, Metall. Trans. A, 1978, 9A(March), p 439-450

19. R.M. Horn and R.O. Ritchie, Mechanisms of Tempered Martensite Embrittlement in Low Alloy Steels, Metall. Trans. A, 1978, 9(8), p $1039-1053$

20. J.P. Materkowski and G. Krauss, Tempered Martensite Embrittlement in SAE 4340 Steel, Metall. Trans. A, 1979, 10(November), p 16431651

21. G. Krauss, Low Toughness and Embrittlement Phenomena in Steels, in Steels: Processing, Structure, and Performance, ASM International, Materials Park, OH, 2005, p 397-398
22. M. Sarikaya, A.K. Jhingan, and G. Thomas, Retained Austenite and Tempered Martensite Embrittlement in Medium Carbon Steels, Metall. Mater. Trans. A, 1983, 14(6), p 1121-1133

23. H. Kwon and C.H. Kim, Tempered Martensite Embrittlement in FeMo-C and Fe-W-C Steel, Metall. Trans. A, 1983, 14(7), p 1389-1394

24. F. Zia-Ebrahimi and G. Krauss, Mechanisms of Tempered Martensite Embrittlement in Medium-Carbon Steels, Acta Metall., 1984, 32(10), p $1767-1777$

25. N. Bandyopadhyay and C.J. McMahon, The Micro-Mechanisms of Tempered Martensite Embrittlement in 4340-Type Steels, Metall. Trans. A, 1981, 14(7), p 1313-1325

26. J. McMahon and G. Thomas, Microstructure and Design of Alloys, in Proc. 3rd Int. Conf. Strength Metals and Alloys, 1973, p 180

27. J.E. King, R.F. Smith, and J.F. Knott, Toughness Variations during the Tempering of a Plain Carbon Martensitic Steel, in 4th International Conference on Fracture, 1977, p 279-286

28. H.K.D.H. Bhadeshia and D.V. Edmonds, Tempered Martensite Embrittlement: Role of Retained Austenite and Cementite, Met. Sci., 1979, 13, p 325-334

29. G.R. Speich and W.C. Leslie, Tempering of Steel, Metall. Trans., 1972, 3(May), p 1043-1054

Publisher's Note Springer Nature remains neutral with regard to jurisdictional claims in published maps and institutional affiliations. 